\title{
Article \\ Risks of Aromatase Inhibitor-Related Cardiotoxicity in Patients with Breast Cancer in Asia
}

\author{
Wei-Ting Chang ${ }^{1,2,3}$, Po-Wei Chen ${ }^{2,4} \mathbb{D}_{\text {, Hui-Wen Lin }}^{4,5}{ }^{\mathbb{D}}$, Yu-Hsuan Kuo ${ }^{6}$, Sheng-Hsiang Lin ${ }^{2,5,7, *,+}$ and \\ Yi-Heng $\mathrm{Li}^{4, *,+}$
}

check for updates

Citation: Chang, W.-T.; Chen, P.-W.; Lin, H.-W.; Kuo, Y.-H.; Lin, S.-H.; Li, Y.-H. Risks of Aromatase InhibitorRelated Cardiotoxicity in Patients with Breast Cancer in Asia. Cancers 2022, 14, 508. https://doi.org/ $10.3390 /$ cancers 14030508 Academic Editor: David Wong

Received: 7 December 2021 Accepted: 18 January 2022 Published: 20 January 2022

Publisher's Note: MDPI stays neutral with regard to jurisdictional claims in published maps and institutional affiliations.

Copyright: (C) 2022 by the authors. Licensee MDPI, Basel, Switzerland. This article is an open access article distributed under the terms and conditions of the Creative Commons Attribution (CC BY) license (https:// creativecommons.org/licenses/by/ $4.0 /)$.
1 Division of Cardiology, Department of Internal Medicine, Chi-Mei Medical Center, Tainan 710402, Taiwan; cmcvecho2@gmail.com

2 Institute of Clinical Medicine, College of Medicine, National Cheng Kung University, Tainan 701401, Taiwan; huntershobow@gmail.com

3 Department of Biotechnology, Southern Taiwan University of Science and Technology, Tainan 710301, Taiwan

4 Department of Internal Medicine, National Cheng Kung University Hospital, College of Medicine, National Cheng Kung University, Tainan 701401, Taiwan; alice882233@gmail.com

5 Biostatistics Consulting Center, National Cheng Kung University Hospital, College of Medicine, National Cheng Kung University, Tainan 701401, Taiwan

6 Division of Oncology, Department of Internal Medicine, Chi-Mei Medical Center, Tainan 710402, Taiwan; beethovan@gmail.com

7 Department of Public Health, College of Medicine, National Cheng Kung University, Tainan 701401, Taiwan

* Correspondence: shlin922@ncku.edu.tw (S.-H.L.); heng@ncku.edu.tw (Y.-H.L.); Tel.: +886-6-2353535 (ext. 5962) (S.-H.L.); +886-6-2353535 (ext. 2389) (Y.-H.L.); Fax: +886-6-2753834 (Y.-H.L.)

+ These authors contributed equally to this work.

Simple Summary: In Asian breast cancer patients, whether the risks of major adverse cardio- and cerebrovascular events (MACCEs) are different between users of aromatase inhibitors (AIs) and selective estrogen receptor modulators (SERMs) remains uncertain. In this nationwide cohort study, the risks of MACCEs were significantly higher in users of SERMs compared with users of AIs in those who were at an old age (above 50 years old) or with advanced cancer stage (stage 3-4). Although the choice of hormone therapies is primarily based on the effectiveness regarding cancer survival, AI treatment should be considered for patients for whom the benefits outweigh the risks.

\begin{abstract}
Background: Despite a preferred endocrine therapy for women with estrogen and progesterone receptor-positive breast cancer, aromatase inhibitors (AIs) have been reported to increase risks of cardiovascular events. Given that breast cancer patients in Asia are younger at diagnosis, it is urgent to investigate this safety concern. Methods: Through the Taiwanese National Cohort, we identified breast cancer patients initiating selective estrogen receptor modulators (SERMs) or AIs from 2010 to 2016. Outcomes includes major adverse cardio- and cerebrovascular events (MACCEs). The average follow-up duration was five years. Results: We identified 16,730 breast cancer patients treated with SERMs and 11,728 receiving AIs. The population was older and had more comorbidities in the AI group than in the SERM group. After adjusting for age, cancer stage, cancer therapies, cardiovascular drugs and comorbidities, despite similar risks of MACCEs between AI and SERM users, the risk of HF was significantly higher in patients treated with SERMs after adjusted mortality as a competing risk. When divided by the age of 50 years, despite a similar MACCEs in the younger population, MACCEs remained significantly higher in the older population who received SERMs. Conclusions: In this Asian cohort, we found that among patients of old age or with advanced cancer stage, the use of SERMs was associated with a higher risk of cardiovascular events than the use of AIs.
\end{abstract}

Keywords: breast cancer; aromatase inhibitors; SERMs; mortality; MACCEs; age; cancer stage 


\section{Introduction}

With the improvement of anticancer therapies, the number of long-term cancer survivors of breast cancer has increased, but survivors face threats of cardiovascular complications, including heart failure (HF) and thromboembolic events [1,2]. Patients with tumors that are estrogen receptor (ER)- and progesterone receptor (PR)-positive are eligible for hormone therapy, including selective estrogen receptor modulators (SERMs) and aromatase inhibitors (AIs) [3,4]. Compared with SERMs, AIs have become the preferred adjuvant treatment for postmenopausal women given favorable clinical outcomes [3,5,6]. Emerging evidence reports a potential risk of major adverse cardio- and cerebrovascular events (MACCEs) with AI use compared with tamoxifen, a most frequently used SERM, use [6-9]. Nevertheless, there are inconsistent findings between randomized controlled trials and observational studies [6,9]. Conversely, patients, especially the elders, treated with tamoxifen were at a higher risk for venous thrombotic events during the first 2 years after exposure. Despite some plausible theories indicating an association between AIs and hyperlipidemia, the biological mechanism of AI-induced cardiotoxicity remains largely unknown $[10,11]$. Additionally, no definitive guidelines specifically suggest the choice of hormone therapies between AIs and SERMs regarding the potential cardiotoxicity in patients with breast cancer [12].

To date, the number of patients diagnosed with breast cancer in Asia has continuously increased [13-15]. In contrast to Western patients with breast cancer, Asian patients are relatively younger at diagnosis [14-16]. This implies that most patients with breast cancer in Asia are diagnosed before menopause and have few cardiovascular risk factors [3]. Most importantly, the majority of previous studies focused on breast cancer patients in the Western world, while only a very limited number of Asian populations were included [13,15]. Therefore, it is crucial to evaluate the incidences and clinical characteristics of AI- and SERM-induced cardiotoxicity in Asia. Herein, using the Taiwan National database, we aimed to compare cardiovascular safety between SERMs and AIs in Asian populations.

\section{Patients and Methods}

\subsection{Study Design}

Through Taiwanese National Health Insurance Research Database (NHIRD) and National Cancer Registry, we identified breast cancer patients initiating hormonal therapy with either SERMs or AIs from 2010 to 2016. The data used in this study were the original claims database for reimbursement of all Taiwanese residents from the NHIRD $[17,18]$. The cohort dataset included age, sex, medications, procedures and all medical diagnoses. To protect patients' privacy, all data were anonymized or pseudonymized. The diagnosis codes in the NHIRD were identified using the International Classification of Diseases, Ninth Revision, Clinical Modification (ICD-9-CM) for cases before 2015 and the International Classification of Diseases, Tenth Revision, Clinical Modification (ICD-10-CM) for cases since 2016. It is also feasible to link and continuously follow up all of the claim's data belonging to the same patient within the NHIRD. This study was approved by our institutional review committee (IRB A-EX-109-021; CV code: 10406-E01) and they granted a waiver of informed consent due to its retrospective nature. We included women who received hormone therapies at least twice within the 365 days after the index date of first diagnosis and had no shift of medications. The exclusion criteria for this study were a history of breast cancer (registry for catastrophic illness patients diagnosed before 2010), previous exposure to SERMs (tamoxifen, toremifene, fulvestrant), gonadotropin-Releasing hormone analogue (goserelin and leuprorelin) or AIs (exemestane, anastrazole and letrazole), incomplete medical records, age less than 18 years, and nonfemale sex. Patients who reached outcomes of mortality or MACCEs were also excluded. In addition, we identified patients with breast cancer of all stages using the nationwide cancer registration system in Taiwan. All comorbid conditions and corresponding treatments starting a year prior to diagnosis were extracted from the National Health Insurance Research Database, as well as medication records of breast cancer diagnosis and treatments. The ICD diagnosis and treatment codes 
were used to identify concomitant medical diseases, medications and procedures (Table S1). Information on age, sex, medical history, concomitant medications within the previous three months, and medications or procedures used during the index admission were captured from the database. The flow chart of this study was displayed in Figure S1.

\subsection{Study Endpoint}

The primary outcome was a composite endpoint of MACCE, which included newonset acute myocardial infarction (AMI), HF, and ischemic stroke (including transient ischemic attack). All patients were followed up from the index date to death or loss to follow-up. Because ICD-9 cm was replaced by ICD-10 cm by the Taiwan National Health Insurance in 2016, both ICD 9 and 10 codes (Supplementary Table S1) were used to identify endpoints in the primary outcome during the follow-up. The median follow-up duration were five years.

\subsection{Statistical Analysis}

Continuous variables are presented as the means \pm standard deviations, and categorical variables are presented as numbers and percentages. Because of the nonrandomized nature of the study, propensity score analysis was performed to minimize any selection bias caused by differences in the clinical characteristics between groups. The propensity score is defined as the probability of exposure to the treatment conditional on a study subject's baseline characteristics. In this study, the propensity score for receiving either SERMs or AIs was computed using multivariate logistic regression analysis, conditional on the covariates including index year, age, cancer stage, anti-cancer therapies (bilateral ovariectomy, anthracyclines, taxanes, 5-fluorouracil, cyclophosphamide), CV medication, including angiotensin- converting enzyme inhibitors/angiotensin receptor blockers (ACEI/ARB), beta blocker, statins, anti-platelet agents, anti-coagulants, digoxin, mineralocorticoid receptor antagonist (MRA), comorbidities, including coronary artery disease, peripheral artery disease, hypertension, diabetes mellitus, hyperlipidemia, valve disease, chronic obstructive pulmonary disease, asthma, atrial fibrillation, chronic kidney disease and end stage renal disease (ESRD). Distributions of the clinical characteristics in the 2 groups were evaluated with the absolute standardized mean difference (ASMD) rather than statistical testing. ASMD was calculated as the mean or proportion of a variable divided by the pooled estimate of the standard deviation of that variable, and an ASMD $<0.1$ indicates a negligible difference between the two groups. A multivariate Cox proportional hazards model with inverse probability of treatment weighting (IPTW) was then used to examine the relationship between the endpoints and different treatments. The same variables used for multivariate logistic regression analysis after propensity score matching were also used in the multivariate Cox model. The HRs and their 95\% CIs were calculated from the Cox models after adjusting for all of these potential confounders. In addition, considering the mortality effects that may reduce the incidence of events, the competing risk approach (subdistribution HR; sHR) was also used to estimate the risk of MACCEs from the Cox regression model after adjusting for all of these potential confounders. A Kaplan-Meier curve was constructed for the primary outcome of MACCE and new-onset HF, and the log-rank test was used to compare the difference between groups. We used the same Cox proportional hazards model to estimate $\mathrm{P}$ values for interactions in the subgroup analysis. SAS 9.4 for Windows (SAS Institute Inc., Cary, NC, USA) was used for all data analyses. 


\section{Results}

3.1. Demographic Characteristics of Breast Cancer Patients Receiving SERMs or AIs

Using the NHIRD from 2010 to 2016, we identified 116152 patients newly diagnosed with breast cancer. Among them, 16,730 patients were treated with SERMs, while 11,728 received AIs (Table 1). The population was older in the AI group than in the SERM group $(62.53 \pm 9.05 \mathrm{y} / \mathrm{o}$ vs. $49.63 \pm 11.55 \mathrm{y} / \mathrm{o})$. In terms of cancer stage and treatment, more patients who received AIs were at an advanced cancer stage (stage III or IV) than those who received SERMS (19.8\% vs. 6.07\%), while more SERM users were treated with anthracyclines $(23.93 \%)$, and AI users were treated with taxanes $(30.98 \%)$. The ratio of bilateral ovariectomy was similar between these two groups (AIs vs. SERMs as $0.63 \%$ vs. $1.13 \%)$. Notably, compared with patients who received SERMs, those receiving AIs had more comorbidities, including hypertension, diabetes, hyperlipidemia and coronary artery disease, and they received more cardiovascular drugs, such as ACEIs/ARBs, statins and antiplatelet and anticoagulant agents. 
Table 1. The baseline characteristics of breast cancer patients treated with either Aromatase inhibitors (AIs) or selective estrogen receptor modulators (SERMs) before and after inverse probability of treatment weighting (IPTW).

\begin{tabular}{|c|c|c|c|c|c|c|c|c|c|c|}
\hline \multirow{3}{*}{ Caption } & \multicolumn{10}{|c|}{ Inverse Probability of Treatment Weighting } \\
\hline & \multicolumn{2}{|c|}{ Total } & \multicolumn{2}{|c|}{ SERMs } & \multicolumn{3}{|c|}{ Before } & SERMs & \multirow{2}{*}{$\begin{array}{l}\text { After } \\
\text { Aromatase Inhibitors } \\
N=\text { Pseudo Data }\end{array}$} & \multirow[b]{2}{*}{ ASMD } \\
\hline & $N=28,458$ & $(\%)$ & $N=16,730$ & $(\%)$ & $N=11,728$ & $(\%)$ & ASMD & $N=$ Pseudo Data & & \\
\hline Age (mean \pm SD) & \multicolumn{2}{|c|}{$54.94 \pm 12.35$} & \multicolumn{2}{|c|}{$49.63 \pm 11.55$} & \multicolumn{2}{|c|}{$62.53 \pm 9.05$} & 1.244 & $61.64 \pm 25.38$ & $59.17 \pm 12.52$ & 0.123 \\
\hline Age (median $\pm \mathrm{IQR})$ & \multicolumn{2}{|c|}{$54 \pm 17$} & \multicolumn{2}{|c|}{$47 \pm 13$} & \multicolumn{2}{|c|}{$61 \pm 12$} & & $60 \pm 31$ & $58 \pm 11$ & \\
\hline Stage & & & & & & & 0.515 & & & 0.101 \\
\hline 0 & 1799 & 6.32 & 1454 & 8.69 & 345 & 2.94 & & 5.40 & 5.68 & \\
\hline 1 & 11,502 & 40.42 & 7558 & 45.18 & 3944 & 33.63 & & 38.01 & 39.46 & \\
\hline 2 & 11,818 & 41.53 & 6702 & 40.06 & 5116 & 43.62 & & 41.32 & 41.75 & \\
\hline 3 & 1986 & 6.98 & 753 & 4.50 & 1233 & 10.51 & & 8.70 & 7.55 & \\
\hline 4 & 1353 & 4.75 & 263 & 1.57 & 1090 & 9.29 & & 6.58 & 5.56 & \\
\hline \multicolumn{11}{|l|}{ Therapies use during } \\
\hline Bilateral ovariectomy & 263 & 0.92 & 189 & 1.13 & 74 & 0.63 & 0.053 & 0.80 & 1.01 & 0.022 \\
\hline Anthracyclines & 6215 & 21.84 & 4004 & 23.93 & 2211 & 18.85 & 0.124 & 17.78 & 19.62 & 0.047 \\
\hline Taxanes & 6733 & 23.66 & 3100 & 18.53 & 3633 & 30.98 & 0.292 & 17.94 & 23.15 & 0.129 \\
\hline 5-fluorouracil & 5973 & 20.99 & 3907 & 23.35 & 2066 & 17.62 & 0.143 & 17.43 & 18.97 & 0.040 \\
\hline Cyclophosphamide & 8071 & 28.36 & 5166 & 30.88 & 2905 & 24.77 & 0.137 & 23.68 & 25.71 & 0.047 \\
\hline \multicolumn{11}{|l|}{ CV medications } \\
\hline ACEI/ARB & 4197 & 14.75 & 1596 & 9.54 & 2601 & 22.18 & 0.351 & 19.60 & 18.65 & 0.024 \\
\hline Beta blocker & 4063 & 14.28 & 1858 & 11.11 & 2205 & 18.80 & 0.217 & 18.20 & 17.38 & 0.021 \\
\hline Statins & 2574 & 9.04 & 898 & 5.37 & 1676 & 14.29 & 0.303 & 11.32 & 11.38 & 0.002 \\
\hline Anti-coagulants & 161 & 0.57 & 55 & 0.33 & 106 & 0.90 & 0.074 & 0.76 & 0.73 & 0.004 \\
\hline Digoxin & 81 & 0.28 & 32 & 0.19 & 49 & 0.42 & 0.041 & 0.46 & 0.41 & 0.008 \\
\hline MRA & 361 & 1.27 & 166 & 0.99 & 195 & 1.66 & 0.059 & 1.14 & 1.34 & 0.018 \\
\hline \multicolumn{11}{|l|}{ Comorbidities } \\
\hline Coronary artery disease & 1459 & 5.13 & 541 & 3.23 & 918 & 7.83 & 0.202 & 7.46 & 6.70 & 0.030 \\
\hline Peripheral artery disease & 250 & 0.88 & 105 & 0.63 & 145 & 1.24 & 0.063 & 0.95 & 1.02 & 0.007 \\
\hline Hypertension & 8264 & 29.04 & 3219 & 19.24 & 5045 & 43.02 & 0.531 & 39.26 & 36.29 & 0.061 \\
\hline Diabetes mellitus & 4124 & 14.49 & 1500 & 8.97 & 2624 & 22.37 & 0.375 & 19.25 & 18.18 & 0.027 \\
\hline Hyperlipidemia & 4723 & 16.60 & 1815 & 10.85 & 2908 & 24.80 & 0.371 & 20.26 & 20.30 & 0.001 \\
\hline Valvular heart disease & 799 & 2.81 & 438 & 2.62 & 361 & 3.08 & 0.028 & 2.74 & 3.06 & 0.019 \\
\hline COPD & 516 & 1.81 & 235 & 1.40 & 281 & 2.40 & 0.073 & 2.49 & 2.27 & 0.015 \\
\hline Asthma & 687 & 2.41 & 361 & 2.16 & 326 & 2.78 & 0.040 & 2.71 & 2.88 & 0.011 \\
\hline Atrial fibrillation & 161 & 0.57 & 57 & 0.34 & 104 & 0.89 & 0.070 & 0.97 & 0.83 & 0.015 \\
\hline Chronic kidney disease & 804 & 2.83 & 287 & 1.72 & 517 & 4.41 & 0.157 & 3.45 & 3.37 & 0.004 \\
\hline ESRD & 16 & 0.06 & 5 & 0.03 & 11 & 0.09 & 0.026 & 0.10 & 0.07 & 0.010 \\
\hline
\end{tabular}

ASMD = absolute standardized mean difference; $\mathrm{CV}$ = cardiovascular; ACEI/ARB = angiotensin-converting enzyme inhibitor/Angiotensin Receptor Blocker; MRA = mineralocorticoidreceptor antagonists; ESRD = end-stage renal disease. 


\subsection{Risks of MACCEs between Breast Cancer Patients Receiving SERMs or AIs}

The crude risks of MACCEs, including HF, AMI and ischemic stroke, were significantly higher among SERM users than among AI users (Table 2). With regard to older ages and more coexisting medical conditions among AI users, ages, cancer stage, cancer therapies, cardiovascular drugs and comorbidities were adjusted. Interestingly, after adjustment, although the differences in the risks of AMI and ischemic stroke diminished between groups, the risk of HF was persistently higher in patients receiving AIs than in patients receiving SERMs (adjusted HR: 0.884; CI: 0.810-0.966, $p=0.007$ ). Furthermore, given a high risk of death among cancer patients, we adjusted the HR with mortality as a competing event, while the sHR of HF remained significantly reduced in AI users compared with SERM users (sHR: 0.885; CI: 0.812-0.966, $p=0.006$ ).

Table 2. The crude and adjusted hazard ratio (HR) of MACCE, AMI, HF and ischemic stroke in users of SERMs and AIs in NHIRD cohort.

\begin{tabular}{|c|c|c|c|c|c|c|c|c|c|}
\hline Caption & $\begin{array}{c}\text { Total } \\
N=28,458\end{array}$ & $\begin{array}{c}\text { SERMs } \\
\text { (Ref.) } \\
N=16,730\end{array}$ & $\begin{array}{l}\text { Aromatase } \\
\text { Inhibitors } \\
N=11,728\end{array}$ & $\begin{array}{c}\text { Crude HR } \\
(95 \% \text { CI })\end{array}$ & $p$ Value & $\begin{array}{c}\text { Adjusted } \\
\text { HR }(95 \% \text { CI })\end{array}$ & $p$ Value & $\begin{array}{l}\text { Adjusted sHR } \\
(95 \% \text { CI) }\end{array}$ & $p$ Value \\
\hline MACCE & 1725 (6.06) & 795 (4.75) & $930(7.93)$ & $\begin{array}{c}0.586 \\
(0.552-0.622)\end{array}$ & $<0.001$ & $\begin{array}{c}0.974 \\
(0.912-1.039)\end{array}$ & 0.423 & $\begin{array}{c}0.970 \\
(0.910-1.035)\end{array}$ & 0.362 \\
\hline $\mathrm{HF}$ & 924 (3.25) & $432(2.58)$ & $492(4.20)$ & $\begin{array}{c}0.518 \\
(0.478-0.562)\end{array}$ & $<0.001$ & $\begin{array}{c}0.884 \\
(0.810-0.966)\end{array}$ & 0.007 & $\begin{array}{c}0.885 \\
(0.812-0.966)\end{array}$ & 0.006 \\
\hline AMI & $110(0.39)$ & $45(0.27)$ & $65(0.55)$ & $\begin{array}{c}0.422 \\
(0.333-0.536)\end{array}$ & $<0.001$ & $\begin{array}{c}1.061 \\
(0.814-1.382)\end{array}$ & 0.662 & $\begin{array}{c}1.015 \\
(0.785-1.311)\end{array}$ & 0.912 \\
\hline $\begin{array}{l}\text { Ischemic } \\
\text { stroke }\end{array}$ & $883(3.10)$ & $406(2.43)$ & $477(4.07)$ & $\begin{array}{c}0.655 \\
(0.602-0.713)\end{array}$ & $<0.001$ & $\begin{array}{c}1.054 \\
(0.961-1.156)\end{array}$ & 0.263 & $\begin{array}{c}1.036 \\
(0.945-1.136)\end{array}$ & 0.450 \\
\hline
\end{tabular}

Model was adjusted for age, stage, therapies use during (bilateral ovariectomy, Anthracyclines, Taxanes, 5fluorouracil, Cyclophosphamide), CV medication (ACEI/ARB, beta blocker, statins, anti-platelet agents, anticoagulants, digoxin, MRA), comorbidities (coronary artery disease, peripheral artery disease, Hypertension, diabetes mellitus, hyperlipidemia, valve disease, COPD, asthma, atrial fibrillation, chronic kidney disease, ESRD) $\mathrm{HR}=$ hazard ratio; $\mathrm{sHR}=$ subdistribution hazard ratio. Abbreviations as Table 1; MACCE = major adverse cardio-cerebral events; $\mathrm{HF}=$ heart failure; $\mathrm{AMI}=$ acute myocardial infarction.

In the five-year follow-up period, the rates free from MACCEs were also lower among AI users than among SERM users (Figure 1A). After one, three and five years from the index date, the rates free from MACCEs were $95.15 \%, 89.25 \%$ and $83.08 \%$, respectively, among SERM users, compared with $97.96 \%, 95.29 \%$ and $92.76 \%$ in AI users. With regard to the different cardiovascular outcomes, the rates of HF (Figure 1B), AMI (Figure 1C) and ischemic stroke (Figure 1D) were persistently higher among patients receiving SERMs than among patients treated with AIs. 
A

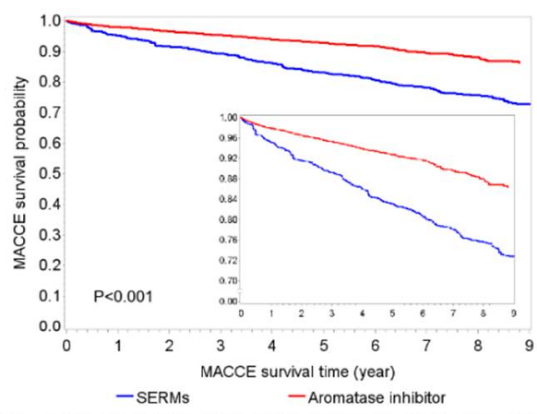

SERMS $2335520894192941761614288115178964 \quad 6257 \quad 3564 \quad 1448$ Aromatase inhibitors $21540206441985117472 \quad 1154968793320 \quad 1395 \quad 776 \quad 244$

C

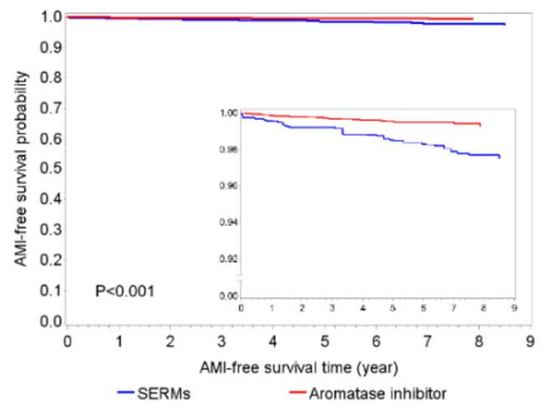

$\begin{array}{lllllll}\text { SERMS } & 2335521527204591904915624 & 12687 & 10089 & 7122 & 4129 & 1721\end{array}$ Aromatase inhibitors $215402101420428181631210872523497 \quad 1510 \quad 845 \quad 276$
B

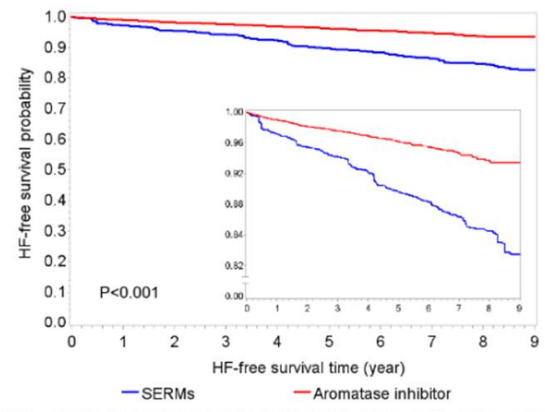

SERMs $23355212862001918453150931215095656684 \quad 3825 \quad 1589$

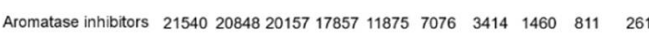

D

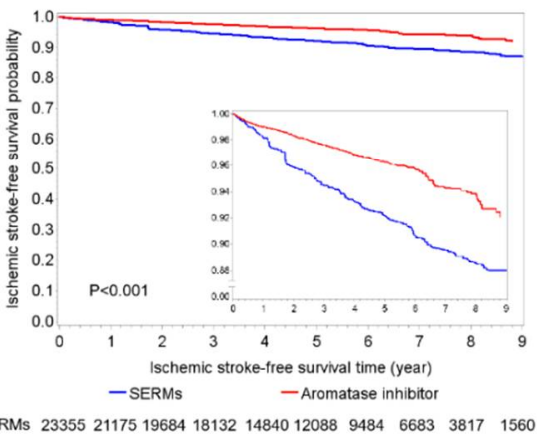

SERMS $2335521175196841813214840120889484 \quad 6683 \quad 3817 \quad 1560$ Aromatase inhibitors $21540208372014517798117897056 \quad 3404 \quad 1445 \quad 809 \quad 257$

Figure 1. The estimated probabilities of patients being free from (A) major adverse cardio-cerebral events (MACCE), (B) heart failure (HF) (C) acute myocardial infarction (AMI) and (D) ischemic stroke among breast cancer patients receiving either selective estrogen receptor modulators (SERMs) or aromatase inhibitors (AIs).

\subsection{Focusing on Breast Cancer Patients at Different Ages}

Given that, to date, the use of AIs is approved only for patients at menopause [1], we further aimed to investigate the effects of AI use on cardiovascular outcomes among patients at different ages. When divided by the age of 50 years old, we found that surprisingly, among young patients ( $<50 \mathrm{y} / \mathrm{o})$ who received AIs, the risks of MACCEs were similar between AI and SERM users (adjusted HR: 0.982; CI: 0.699-1.381, $p=0.919$ ) (Table 3). After adjusting the HR with mortality as a competing event, the risk of MACCEs remained insignificantly different between groups (sHR: 0.976; CI: 0.693-1.372, $p=0.887$ ). In contrast, when focusing on patients aged above $50 \mathrm{y} / \mathrm{o}$, the risk of MACCEs remained lower among AI users compared with SERM users (adjusted HR: 0.798; CI: 0.746-0.854, $p<0.001$ ). In terms of each cardiovascular endpoint, there were significant reductions in the risks of HF (adjusted HR: 0.741; CI: 0.677-0.812, $p<0.001$ ), AMI (adjusted HR: 0.749; CI: 0.578-0.971, $p=0.029$ ) and ischemic stroke (adjusted HR: 0.877; CI: 0.797-0.965, $p=0.007$ ) among AI users compared with SERM users. The abovementioned risks were persistently lower among AI users after adjusting for mortality as a competing risk. 
Table 3. The crude and adjusted hazard ratio (HR) of MACCE, AMI, HF and ischemic stroke in users of SERMs and AIs in the young $(<50 \mathrm{y} / \mathrm{o})$ and aged $(\geqq 50 \mathrm{y} / \mathrm{o})$ population.

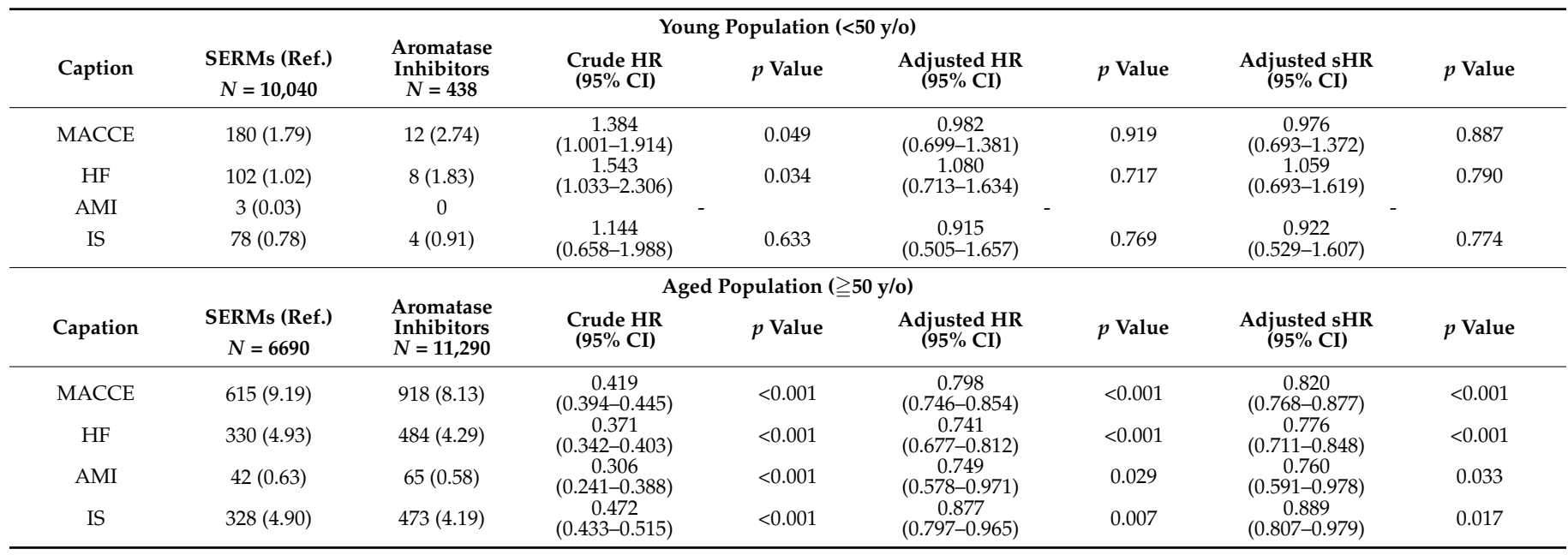

Abbreviations as Tables 1 and 2. ASMD $=$ absolute standardized mean difference; $\mathrm{CV}=$ cardiovascular; $\mathrm{ACEI} / \mathrm{ARB}$ $=$ angiotensin-converting enzyme inhibitor $/$ Angiotensin Receptor Blocker; MRA = mineralocorticoid-receptor antagonists; $\mathrm{ESRD}=$ end-stage renal disease. $\mathrm{HR}=$ hazard ratio; $\mathrm{sHR}=$ subdistribution hazard ratio. Abbreviations as Table $1 ; \mathrm{MACCE}=$ major adverse cardio-cerebral events; $\mathrm{HF}=$ heart failure; $\mathrm{AMI}=$ acute myocardial infarction.

\subsection{Focusing on Breast Cancer Patients with Different Cancer Stages}

According to emerging evidence, the benefits of hormone therapy in reducing the risk of breast cancer recurrence and progression seem to be independent of cancer stage [1]. Nevertheless, whether the effects of AI on cardiovascular outcomes are associated with cancer stages remains uncertain. We further divided the study patients into relatively early (stage 0-2) and advanced cancer stages (stage 3-4) and observed that in patients with early cancer stages, the risks of MACCEs (adjusted HR: 0.930; CI: 0.862-1.002, $p=0.058$ ) were insignificantly different between AI and SERM users (Table 4). Despite a significant risk reduction in AMI among AI users (adjusted HR: 0.54; CI: 0.397-0.735, $p<0.001$ ), the phenomenon diminished in terms of the outcomes of HF (adjusted HR: 0.910; CI: 0.821-1.009, $p=0.074$ ) and ischemic stroke (adjusted HR: 0.968; CI: 0.873-1.073, $p=0.537$ ) compared with the risks among SERM users. In contrast, focusing on patients with advanced cancer stage, there were significantly reduced risks of MACCEs (adjusted HR: 0.492; CI: 0.425-0.569, $p<0.001$ ) among AI users compared with SERM users. Similarly, the risks of HF (adjusted HR: 0.470; CI: 0.391-0565, $p<0.001$ ) and ischemic stroke (adjusted HR: 0.434; CI: 0.337-0.559, $p<0.001$ ) were also lower among patients treated with AIs than among those treated with SERMs, but in contrast, the risk of AMI increased (adjusted HR: 2.567; CI: 1.366-4.827, $p=0.003$ ). 
Table 4. The crude and adjusted hazard ratio (HR) of MACCE, AMI, HF and ischemic stroke in users of SERMs and AIs in breast cancer patients at different cancer stages.

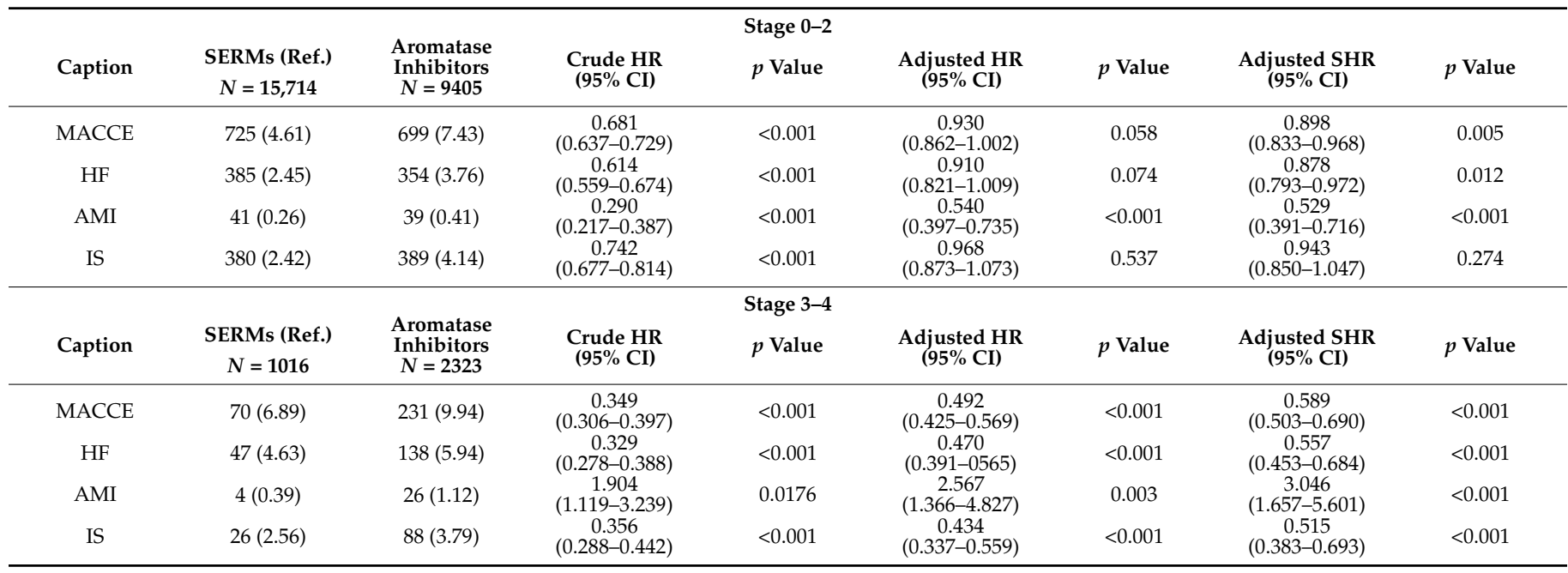

Abbreviations as Tables 1 and 2. ASMD $=$ absolute standardized mean difference; $\mathrm{CV}=$ cardiovascular; ACEI/ARB $=$ angiotensin-converting enzyme inhibitor/Angiotensin Receptor Blocker; MRA = mineralocorticoid-receptor antagonists; $\mathrm{ESRD}=$ end-stage renal disease. $\mathrm{HR}=$ hazard ratio; $\mathrm{sHR}=$ subdistribution hazard ratio. Abbreviations as Table 1; MACCE = major adverse cardio-cerebral events; $\mathrm{HF}$ = heart failure; $\mathrm{AMI}=$ acute myocardial infarction.

\subsection{Subgroup Analysis of Hormone Therapy-Related MACCEs}

Although AI use was associated with reduced risks of MACCEs in patients with old age or advanced cancer stage, in the subgroup analysis, we found that patients with a cumulative course of anthracycline, taxanes or cyclophosphamide exhibited a higher risk of MACCEs among AI users than SERM users (Figure 2). Additionally, among patients with cardiovascular diseases or risk factors, including CAD, DM or CKD, although insignificant, there was a trend of higher risks of MACCEs regarding AI use compared with SERM use. Conversely, patients free from cardiovascular risk factors or concomitant chemotherapy with potential cardiotoxicity were prone to having reduced risks of MACCEs using AIs instead of SERMs.

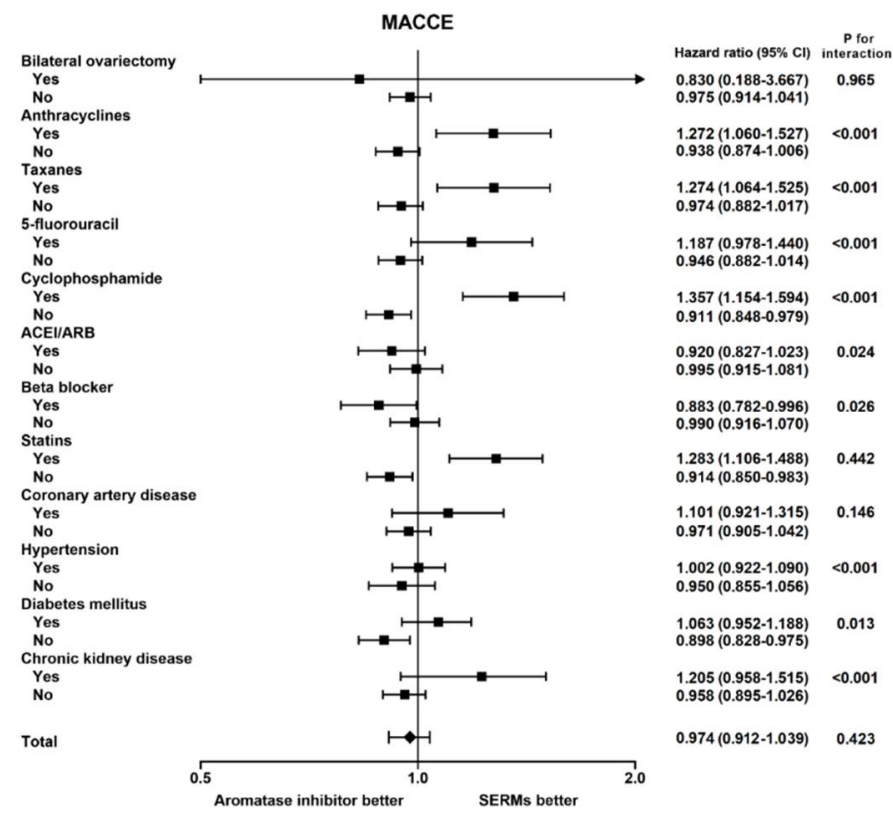

Figure 2. The subgroup analysis of risks of major adverse cardio-cerebral events (MACCEs) in breast cancer patients receiving either selective estrogen receptor modulators (SERMs) or Aromatase inhibitors (AIs) in the nationwide cohort. 


\section{Discussion}

In this Asian cohort, interestingly, we found that the risk of HF was lower in patients treated with AIs than in those treated with SERMs. When focusing on patients younger than 50 years old, the phenomenon diminished but remained significant in the older population. Similarly, in patients with advanced cancer stage, the risks of MACCEs were reduced among those who were treated with AIs. Collectively, in contrast to previous studies that showed a higher cardiovascular risk of AI use than SERM use [6,7,9], in this Asian cohort, we found that the use of AIs was associated with a lower risk of cardiovascular events than the use of SERMs among breast cancer patients in old age or at an advanced cancer stage. To our knowledge, this is the first observational study focusing on the effects of hormone therapies in Asian patients with breast cancer.

Previous studies indicated a benefit of AIs in cancer-free survival compared with tamoxifen [3,19]. The results from the Early Breast Cancer Trialists' Collaborative Group (EBCTCG) showed that AIs reduce recurrence rates by approximately 30\% compared with tamoxifen [5]. Nevertheless, subsequent meta-analyses and randomized controlled trials (RCTs) demonstrated that AIs are associated with an increased risk of ischemic events compared with tamoxifen $[6,9,20]$. Similarly, in the trial of Breast International Group, which focused on letrozole, a type of AI, there was a significantly increased risk of HF ( $0.65 \%$ vs. $0.32 \%$ in letrozole vs. tamoxifen) [21]. Khosrow-Khavar et al., reported that AI use was observed to increase the risks of HF and cardiovascular mortality compared with tamoxifen [9]. In contrast, observational studies reported inconsistent findings. Haque et al., using the Kaiser Permanente health insurance database, did not find that AIs increased risks of cardiac ischemia, stroke, HF or cardiomyopathy among women without a history of cardiovascular disease [6]. Similarly, using HealthCore Integrated Research Databases, Ligibel et al., did not observe an association between the use of AIs and AMI among women above 67 years old compared with tamoxifen use [22]. Another observational cohort study in Spain showed that AI use lowered $20 \%$ of all-cause mortality compared with tamoxifen use without increasing the risk of cardiovascular and thromboembolic events [20]. The authors even suggested that AI therapy should be the upfront option in adjuvant hormonal therapy [20]. Owing to the heterogeneity of these trials, including the study design and included populations, to date, the inconsistent findings have led to inconclusive results regarding the cardiovascular risks of AIs. Notably, the decision to initiate therapies with either AIs or SERMs mainly depends on the hormone receptor status instead of cardiovascular risk profiles [3,5]. Given that AI use is currently approved for postmenopausal women only, the aged population per se is at a higher cardiovascular risk [3,23]. Additionally, most patients under AI use concomitantly receive adjuvant chemotherapies with potential cardiotoxicity $[8,24]$. All abovementioned factors may contribute to unfavorable cardiovascular outcomes that were previously reported in patients receiving AIs. Most importantly, to date, there was no guideline specifically recommending the choice between $\mathrm{AI}$ and SERMs based on the cardiovascular risks of patients with breast cancer [12]. Consensus statement between oncologists and cardiologists mainly focused on the risk management and early detecting of cardiotoxicities [12].

Previous observational studies indicated an ethnic gradient in terms of the frequency of thromboembolism, and Asians have the lowest risk compared with other races $[13,25,26]$. Therefore, Asian women with breast cancer have unique disease characteristics and should be treated specifically. However, studies investigating the correlation between hormone therapies and cardiovascular risks in Asian women are scant and limited regarding the use of SERMs. A recently published study in Korea using a National Health Information Database focused on breast cancer patients above 55 years old and found a protective effect of tamoxifen against cardiovascular events that is absent with AIs [7]. Another study focusing on Taiwanese women with AMI also observed a 1.71 times higher risk among tamoxifen users than among nonusers [27]. However, using the Taiwan population database, Chen et al., reported no increased venous thromboembolism risk in breast cancer patients receiving adjuvant tamoxifen [28]. In contrast, our study is the first comparing 
the cardiovascular risks between the use of AIs and SERMs in Asian women with breast cancer and revealed a potential lower risk of MACC outcomes in AI users, especially in the aged population. Ethnicity differences should be considered as physicians choose optimal endocrine treatments for breast cancer patients.

Although AI use has been proposed to increase cardiovascular events by increasing low-density lipoprotein cholesterol levels, RCTs have not observed significant changes in cholesterol levels in patients receiving AIs [21,29]. In contrast, a plausible cardioprotective effect of tamoxifen has been reported based on its ability to lower serum lipid levels [30,31]. Nevertheless, SERM may have a different impact on lipid profiles in preand postmenopausal women. Although beneficial alterations of lipid metabolism were observed in premenopausal breast cancer patients treated with tamoxifen [11], in postmenopausal patients with breast cancer, marked hypertriglyceridemia was observed after tamoxifen treatment [10]. On the other hand, evidence from the Breast International Group (BIG) and Tamoxifen and Exemestane in Women With Postmenopausal Early Breast Cancer (TEAM) showed that SERMs may stimulate platelet activation and result in thromboembolic events [32-34]. Beyond the evidence regarding hormone therapy on thrombosis, the effect of either AIs or SERMs on myocardial function is largely unknown. Despite a proposed cardioprotective role of estrogen in females, the effects of hormone suppression or replacement therapies on cardiovascular outcomes remain controversial $[35,36]$. Using the UK Clinical Practice Research Datalink, it has been reported that AI use was associated with an increased risk of HF and cardiovascular death compared with tamoxifen use [9]. Conversely, in this Asian cohort, we found that HF was lower in patients treated with AIs than in those treated with SERMs. In contrast to SERMs, AIs play pivotal roles in balancing androgen and estrogen. Using a genetic model of aromatase tissue-deficient (ArKO) female mice, Bell et al. found that isolated ArKO cardiomyocytes exposed to a high $\mathrm{Ca} 2+$ load exhibited greater $\mathrm{Ca} 2+$ transient and contractile amplitudes [37]. Their findings suggested that by suppressing aromatase in females, a relative withdrawal of estrogen may support myocardial inotropes via optimized Ca2+ handling in response to stress [37]. However, whether AIs have a positive or negative impact on cardiac functions requires more investigation.

In terms of the germline genetic polymorphism, through comparing Asia Breast Cancer Consortium (ABCC) and another European Breast Cancer Association Consortium (BCAC), a meta-analysis identifying thirty-one novel breast cancer susceptibility loci unique for Asian women [38]. Additionally, the VGH-TAYLOR study is designed to understand the genetic profiling of different subtypes of breast cancer in Taiwan and define the molecular risk factors for breast cancer recurrence. Although using Genome-wide association study (GWAS), Park et al., studied genetic variants related to anthracycline-induced cardiotoxicity in early breast cancer in Korea, it remains lacking a study focusing on genetic polymorphism in the development of cardiotoxicities among breast cancer patients receiving hormone therapies including AIs and SERMs [39]. Nevertheless, genetic variability could be a possible explanation of this different effect of hormone therapies in Asian populations compared with the Western ones, but more studies are required.

In our subgroup analysis, we found that among patients younger than 50 years old, the risks of MACCEs among AI users were similar compared with SERM users but were significantly lower in the older population. Given that, in Asia, a large portion of breast cancer patients are diagnosed before menopause, which is different from most observational studies $[7,9,29]$, this cohort provides additional information regarding the effects of AI use on the younger population. Our findings also echo that AI use should be preserved for patients above 50 years old. In terms of cancer stage, although a metaanalysis of the Arimidex, Tamoxifen, Alone or in Combination and Breast International Group clinical trials indicated that AI use was more favorable than tamoxifen use in almost all circumstances regardless of breast cancer stage [40], in this cohort, we found that in patients with advanced breast cancer stages (stage 3-4), the risks of MACCEs were reduced most significantly among AI users compared with SERM users. However, 
among patients who were concomitantly or sequentially treated with chemotherapies with potential cardiotoxicities, AI use was associated with a higher risk of MACCE than SERM use. Therefore, the decision between AIs and SERMs should be tailored according to an individual's age, cancer stage and characteristics.

Our study is not devoid of limitations. First, due to its retrospective study design, patients were not randomized to receive either SERMs or AIs. Even though the study was designed under a matching method to reduce the difference between the two groups, there was still selection bias due to patients' menstrual status and physical conditions. To compensate for this limitation, in the subgroups analysis we divided the studied population by the age of 50 years and further investigated the impact of AI uses on cardiovascular health. Additionally, data regarding smoking, physical activity and body mass index, which may be also associated cardiovascular risks, were not available in this study.

\section{Conclusions}

Collectively, using this nationwide Asian cohort, we observed a higher risk of MACCEs in breast cancer patients receiving SERMs than those receiving AIs, especially at ages above 50 years or having advanced cancer stages. Although the choice of hormone therapies in breast cancer patients is primarily based on their effectiveness against cancer survival, therapy-associated cardiovascular burdens remain a concern influencing longterm morbidities and quality of life. Hereby, we provide evidence regarding the role of AIs in upfront adjuvant hormonal therapy, while more investigations, especially RCTs, are mandatory to support our findings.

Supplementary Materials: The following supporting information can be downloaded at: https: / / www.mdpi.com/article/10.3390 / cancers14030508/s1, Figure S1: The algorithm of study design; Table S1: Lists of ICD 9 and 10 codes; Table S2: The causes of death among the studies population.

Author Contributions: Conceptualization, W.-T.C., S.-H.L., Y.-H.L., H.-W.L. and Y.-H.K.; methodology, W.-T.C., S.-H.L. and H.-W.L.; software, S.-H.L. and H.-W.L.; validation, W.-T.C., S.-H.L., Y.-H.L., H.-W.L., P.-W.C. and Y.-H.K.; formal analysis, S.-H.L. and H.-W.L.; investigation, W.-T.C., S.-H.L., Y.-H.L., H.-W.L., P.-W.C., Y.-H.K.; resources, Y.-H.L.; data curation, S.-H.L. and H.-W.L.; writing—original draft preparation, W.-T.C.; writing—review and editing, W.-T.C., Y.-H.L., H.-W.L.; visualization, W.-T.C., S.-H.L., Y.-H.L., H.-W.L., P.-W.C., Y.-H.K.; supervision, S.-H.L. and Y.-H.L.; project administration, W.-T.C. and Y.-H.L.; funding acquisition, W.-T.C. and Y.-H.L. All authors have read and agreed to the published version of the manuscript.

Funding: This study is supported by National Cheng Kung University Hospital and Chi-Mei Medical Center. Wei-Ting Chang is granted by Ministry of Science and Technology (MOST 109-2326-B-384001-MY3).

Institutional Review Board Statement: The study was conducted according to the guidelines of the Declaration of Helsinki and approved by National Cheng Kung University Hospital and Chi-Mei Medical Center, Tainan, Taiwan (IRB A-EX-109-021; CV code: 10406-E01).

Informed Consent Statement: Not applicable.

Data Availability Statement: Given that data is from a publicly accessible repository 3rd Party, NHIRD, data sharing is not applicable.

Acknowledgments: We are grateful to Health Data Science Center, National Cheng Kung University Hospital for providing administrative and technical support.

Conflicts of Interest: The authors declare no conflict of interest.

\section{References}

1. Zamorano, J.L.; Lancellotti, P.; Rodriguez Munoz, D.; Aboyans, V.; Asteggiano, R.; Galderisi, M.; Habib, G.; Lenihan, D.J.; Lip, G.Y.H.; Lyon, A.R.; et al. 2016 ESC Position Paper on cancer treatments and cardiovascular toxicity developed under the auspices of the ESC Committee for Practice Guidelines: The Task Force for cancer treatments and cardiovascular toxicity of the European Society of Cardiology (ESC). Eur. Heart J. 2016, 37, 2768-2801. [CrossRef] [PubMed] 
2. Chang, H.-M.; Moudgil, R.; Scarabelli, T.; Okwuosa, T.M.; Yeh, E.T.H. Cardiovascular Complications of Cancer Therapy: Best Practices in Diagnosis, Prevention, and Management: Part 1. J. Am. Coll. Cardiol. 2017, 70, 2536-2551. [CrossRef]

3. Josefsson, M.L.; Leinster, S.J. Aromatase inhibitors versus tamoxifen as adjuvant hormonal therapy for oestrogen sensitive early breast cancer in post-menopausal women: Meta-analyses of monotherapy, sequenced therapy and extended therapy. Breast 2010, 19, 76-83. [CrossRef]

4. Jacobse, J.N.; Schaapveld, M.; Boekel, N.B.; Hooning, M.J.; Jager, A.; Baaijens, M.H.A.; Hauptmann, M.; Russell, N.S.; Rutgers, E.J.T.; Aleman, B.M.P.; et al. Risk of heart failure after systemic treatment for early breast cancer: Results of a cohort study. Breast Cancer Res. Treat. 2021, 185, 205-214. [CrossRef] [PubMed]

5. Early Breast Cancer Trialists' Collaborative Group. Aromatase inhibitors versus tamoxifen in early breast cancer: Patient-level meta-analysis of the randomised trials. Lancet 2015, 386, 1341-1352. [CrossRef]

6. Haque, R.; Shi, J.; Schottinger, J.E.; Chung, J.; Avila, C.; Amundsen, B.; Hauptmann, M.; Russell, N.S.; Rutgers, E.J.T.; Aleman, B.M.P. Cardiovascular Disease After Aromatase Inhibitor Use. JAMA Oncol. 2016, 2, 1590-1597. [CrossRef]

7. Choi, S.H.; Kim, K.-E.; Park, Y.; Ju, Y.W.; Jung, J.-G.; Lee, E.S.; Lee, H.-B.; Han, W.; Noh, D.-Y.; Yoon, H.-J.; et al. Effects of tamoxifen and aromatase inhibitors on the risk of acute coronary syndrome in elderly breast cancer patients: An analysis of nationwide data. Breast 2020, 54, 25-30. [CrossRef] [PubMed]

8. Xu, X.; Chlebowski, R.T.; Shi, J.; Barac, A.; Haque, R. Aromatase inhibitor and tamoxifen use and the risk of venous thromboembolism in breast cancer survivors. Breast Cancer Res. Treat. 2019, 174, 785-794. [CrossRef] [PubMed]

9. Khosrow-Khavar, F.; Filion, K.B.; Bouganim, N.; Suissa, S.; Azoulay, L. Aromatase Inhibitors and the Risk of Cardiovascular Outcomes in Women With Breast Cancer: A Population-Based Cohort Study. Circulation 2020, 141, 549-559. [CrossRef]

10. Hozumi, Y.; Kawano, M.; Saito, T.; Miyata, M. Effect of tamoxifen on serum lipid metabolism. J. Clin. Endocrinol. Metab. 1998, 83, 1633-1635. [CrossRef]

11. Love, R.R.; Newcomb, P.A.; Wiebe, D.A.; Surawicz, T.S.; Jordan, V.C.; Carbone, P.P.; DeMets, D.L. Effects of tamoxifen therapy on lipid and lipoprotein levels in postmenopausal patients with node-negative breast cancer. J. Natl. Cancer Inst. 1990, 82, 1327-1332. [CrossRef] [PubMed]

12. Okwuosa, T.M.; Morgans, A.; Rhee, J.-W.; Reding, K.W.; Maliski, S.; Plana, J.-C.; Volgman, A.S.; Moseley, K.F.; Porter, C.B.; Ismail-Khan, R. Impact of Hormonal Therapies for Treatment of Hormone-Dependent Cancers (Breast and Prostate) on the Cardiovascular System: Effects and Modifications: A Scientific Statement From the American Heart Association. Circ. Genom. Precis. Med. 2021, 14, e000082. [CrossRef]

13. Kim, Y.; Yoo, K.-Y.; Goodman, M.T. Differences in incidence, mortality and survival of breast cancer by regions and countries in Asia and contributing factors. Asian Pac. J. Cancer Prev. 2015, 16, 2857-2870. [CrossRef] [PubMed]

14. Leong, S.P.L.; Shen, Z.-Z.; Liu, T.-J.; Agarwal, G.; Tajima, T.; Paik, N.-S.; Sandelin, K.; Derossis, A.; Cody, H.; Foulkes, W.D. Is breast cancer the same disease in Asian and Western countries. World J. Surg. 2010, 34, 2308-2324. [CrossRef]

15. Youlden, D.R.; Cramb, S.M.; Yip, C.H.; Baade, P.D. Incidence and mortality of female breast cancer in the Asia-Pacific region. Cancer Biol. Med. 2014, 11, 101-115. [CrossRef] [PubMed]

16. Wong, F.Y.; Tham, W.Y.; Nei, W.L.; Lim, C.; Miao, H. Age exerts a continuous effect in the outcomes of Asian breast cancer patients treated with breast-conserving therapy. Cancer Commun. 2018, 38, 39. [CrossRef] [PubMed]

17. Cheng, C.-L.; Kao, Y.-H.Y.; Lin, S.-J.; Lee, C.-H.; Lai, M.L. Validation of the National Health Insurance Research Database with ischemic stroke cases in Taiwan. Pharmacoepidemiol. Drug Saf. 2011, 20, 236-242. [CrossRef]

18. Huang, K.; Lin, F.J.; Ou, H.T.; Hsu, C.N.; Huang, L.Y.; Wang, C.C.; Toh, S. Building an active medical product safety surveillance system in Taiwan: Adaptation of the U.S. Sentinel System common data model structure to the National Health Insurance Research Database in Taiwan. Pharmacoepidemiol. Drug Saf. 2021, 30, 97-101. [CrossRef] [PubMed]

19. Torjesen, I. Extra five years of aromatase inhibitors increases disease-free survival in breast cancer. BMJ 2016, 353, i3153. [CrossRef]

20. Pineda-Moncusí, M.; Garcia-Giralt, N.; Diez-Perez, A.; Tusquets, I.; Servitja, S.; Albanell, J.; Prieto-Alhambra, D.; Nogués, X. Thromboembolic, cardiovascular and overall mortality risks of aromatase inhibitors, compared with tamoxifen treatment: An outpatient-register-based retrospective cohort study. Ther. Adv. Med. Oncol. 2020, 12, 1758835920909660. [CrossRef]

21. Mouridsen, H.; Keshaviah, A.; Coates, A.S.; Rabaglio, M.; Castiglione-Gertsch, M.; Sun, Z.; Thürlimann, B.; Mauriac, L.; Forbes, J.F.; Paridaens, R.; et al. Cardiovascular adverse events during adjuvant endocrine therapy for early breast cancer using letrozole or tamoxifen: Safety analysis of BIG 1-98 trial. J. Clin. Oncol. 2007, 25, 5715-5722. [CrossRef]

22. Ligibel, J.A.; O'Malley, J.A.; Fisher, M.; Daniel, G.W.; Winer, E.P.; Keating, N.L. Risk of myocardial infarction, stroke, and fracture in a cohort of community-based breast cancer patients. Breast Cancer Res. Treat. 2012, 131, 589-597. [CrossRef] [PubMed]

23. Meng, J.; Wang, X.; Guan, Y.; Zhang, D. Aromatase inhibitors plus ovarian function suppression versus tamoxifen plus ovarian function suppression for premenopausal women with early stage breast cancer: A systematic review and meta-analysis. Ann. Palliat. Med. 2020, 9, 2294-2302. [CrossRef] [PubMed]

24. Buch, K.; Gunmalm, V.; Andersson, M.; Schwarz, P.; Brøns, C. Effect of chemotherapy and aromatase inhibitors in the adjuvant treatment of breast cancer on glucose and insulin metabolism-A systematic review. Cancer Med. 2019, 8, 238-245. [CrossRef] [PubMed]

25. White, R.H.; Keenan, C.R. Effects of race and ethnicity on the incidence of venous thromboembolism. Thromb. Res. 2009, 123 (Suppl. 4), S11-S17. [CrossRef]

26. Goldhaber, S.Z. Race and venous thromboembolism: Nature or nurture? Circulation 2014, 129, 1463-1465. [CrossRef] 
27. Lai, S.-W.; Lin, C.-L.; Liao, K.-F. Association between tamoxifen use and acute myocardial infarction in women with breast cancer. Medicine 2019, 98, e13925. [CrossRef]

28. Chen, T.W.-W.; Chen, H.-M.; Lin, C.-H.; Huang, C.-S.; Cheng, A.-L.; Lai, M.-S.; Lu, Y.-S. No increased venous thromboembolism risk in Asian breast cancer patients receiving adjuvant tamoxifen. Breast Cancer Res. Treat. 2014, 148, 135-142. [CrossRef]

29. Markopoulos, C.; Chrissochou, M.; Michailidou, A.; Tzoracoleftherakis, E.; Xepapadakis, G.; Papadiamantis, J.; Misitzis, J.; Zobolas, V.; Bafaloukos, D.; Gogas, H. Effect of exemestane on the lipidemic profile of post-menopausal operable breast cancer patients following 5-7 years of adjuvant tamoxifen: Preliminary results of the ATENA substudy. Anticancer Drugs 2005, 16, 879-883. [CrossRef]

30. Joensuu, H.; Holli, K.; Oksanen, H.; Valavaara, R. Serum lipid levels during and after adjuvant toremifene or tamoxifen therapy for breast cancer. Breast Cancer Res. Treat. 2000, 63, 225-234. [CrossRef]

31. Montagnani, A.; Gonnelli, S.; Cadirni, A.; Caffarelli, C.; Del Santo, K.; Pieropan, C.; Campagna, M.; Montomoli, M.; Petrioli, R.; Nuti, R. The effects on lipid serum levels of a 2-year adjuvant treatment with exemestane after tamoxifen in postmenopausal women with early breast cancer. Eur. J. Intern. Med. 2008, 19, 592-597. [CrossRef]

32. Chang, Y.; Lee, J.J.; Chen, W.F.; Chou, D.S.; Huang, S.-Y.; Sheu, J.R. A novel role for tamoxifen in the inhibition of human platelets. Transl. Res. 2011, 157, 81-91. [CrossRef]

33. Colleoni, M.; Giobbie-Hurder, A.; Regan, M.M.; Thürlimann, B.; Mouridsen, H.; Mauriac, L.; Forbes, J.F.; Paridaens, R.; Láng, I.; Smith, I.; et al. Analyses adjusting for selective crossover show improved overall survival with adjuvant letrozole compared with tamoxifen in the BIG 1-98 study. J. Clin. Oncol. 2011, 29, 1117-1124. [CrossRef] [PubMed]

34. van de Velde, C.J.; Rea, D.; Seynaeve, C.; Putter, H.; Hasenburg, A.; Vannetzel, J.-M.; Paridaens, R.; Markopoulos, C.; Hozumi, Y.; Hille, E.T.; et al. Adjuvant tamoxifen and exemestane in early breast cancer (TEAM): A randomised phase 3 trial. Lancet 2011, 377, 321-331. [CrossRef]

35. Abd El-Lateef, S.M.; El-Sayed, E.M.; Mansour, A.M.; Salama, S.A. The protective role of estrogen and its receptors in gentamicininduced acute kidney injury in rats. Life Sci. 2019, 239, 117082. [CrossRef]

36. Iorga, A.; Cunningham, C.M.; Moazeni, S.; Ruffenach, G.; Umar, S.; Eghbali, M. The protective role of estrogen and estrogen receptors in cardiovascular disease and the controversial use of estrogen therapy. Biol. Sex Differ. 2017, 8, 33. [CrossRef] [PubMed]

37. Bell, J.R.; Bernasochi, G.B.; Wollermann, A.C.; Raaijmakers, A.J.; Boon, W.C.; Simpson, E.R.; Curl, C.L.; Mellor, K.; Delbridge, L.M.D. Myocardial and cardiomyocyte stress resilience is enhanced in aromatase-deficient female mouse hearts through CaMKIIdelta activation. Endocrinology 2015, 156, 1429-1440. [CrossRef] [PubMed]

38. Shu, X.; Long, J.; Cai, Q.; Kweon, S.-S.; Choi, J.-Y.; Kubo, M.; Park, S.K.; Bolla, M.K.; Dennis, J.; Wang, Q.; et al. Identification of novel breast cancer susceptibility loci in meta-analyses conducted among Asian and European descendants. Nat. Commun. 2020, 11, 1217. [CrossRef] [PubMed]

39. Park, B.; Sim, S.H.; Lee, K.S.; Kim, H.J.; Park, I.H. Genome-wide association study of genetic variants related to anthracyclineinduced cardiotoxicity in early breast cancer. Cancer Sci. 2020, 111, 2579-2587. [CrossRef]

40. Baum, M. The ATAC (Arimidex, Tamoxifen, Alone or in Combination) adjuvant breast cancer trial in postmenopausal patients: Factors influencing the success of patient recruitment. Eur. J. Cancer 2002, 38, 1984-1986. [CrossRef] 